\title{
Electrocardiogram Changes in Intracranial Space Occupying Lesion
}

\author{
Bhagya R. Jena ${ }^{1}$ Sirivella P. Kumar ${ }^{2}$ \\ ${ }^{1}$ Department of Neuroanesthesiology and Critical Care, All India \\ Institute of Medical Sciences, New Delhi, India \\ ${ }^{2}$ Depatment of Anesthesiology, Critical Care and Pain Medicine, \\ All India Institute of Medical Sciences, New Delhi, India
}

Ashish Bindra ${ }^{1}$

J Neuroanaesthesiol Crit Care:2021;8:79-80

Electrocardiogram (ECG) is an important tool to diagnose and manage different cardiac conditions. At times, ECG changes are reflective of intracranial pathology causing myocardial stress. In such instances, relying and treating cardiac condition alone can be misleading. Intracranial pathologies such as hemorrhage, aneurysm, stroke, injury, or a space-occupying lesion, have been reported to cause ECG changes mimicking acute coronary syndrome. ${ }^{1-3}$ These changes are poorly understood and thought to occur due to altered sympathetic tone and associated catecholamine surge. ${ }^{2}$ Though there are reports mentioning the appearance of ECG changes during acute neurological stress, this is an interesting case wherein an American Society of Anesthesiologists (ASA) grade 1 patient with mass effect came for elective surgery developed ECG abnormalities despite her normal physical status and no suggestive history. The common reported ECG findings mimic acute coronary syndrome with ST elevation and T inversion with signs and symptoms of neurogenic stunned myocardium such as chest pain, shortness of breath, and hemodynamic instability.

A 42-year-old female patient, ASA1 with right sphenoid wing meningioma was admitted for elective craniotomy and tumor excision. There was no history of past illness, orthopnea or dyspnea on exertion, or any other chronic illness. She was on oral phenytoin $300 \mathrm{mg}$ from past 1 week. Other systemic and physical eaxmination was unremarkable. Serum electrolytes and hematological and biochemical investigations were within normal limits. MRI showed dura base enhancing lesion with significant mass effect ( - Fig. 1). Preoperative ECG showed ST depressions with T wave inversions in lead II, V1-V6 (-Fig. 2A). In view of significant ECG changes, preoperative echocardiography was done which did not reveal any significant pathology. Troponin T (Trop T) was negative.

Since the tumor was big, patient was planned for an elective surgery and induction was done with intravenous fentanyl $150 \mu \mathrm{g}$, propofol $120 \mathrm{mg}$, rocuronium $50 \mathrm{mg}$, and a 7.5-mm cuffed polyvinyl chloride tube was used for tracheal intubation. Surgery was done in supine position with

Published online

October 23, 2020
DOI https://doi.org/

$10.1055 / \mathrm{s}-0039-1697217$

ISSN 2348-0548.
Niraj Kumar ${ }^{1}$

Address for correspondence Ashish Bindra, MD, DM, Department of Neuroanesthesiology and Critical Care, All India Institute of Medical Sciences, New Delhi 110029, India (e-mail: dr_ashi2208@yahoo.com).

vigilant intraoperative vital monitoring including 5-lead ECG, heart rate, pulse oximetry, arterial blood pressure, central venous pressure, urine output, and temperature. Significant ST depression persisted in chest leads before induction and continued to be present throughout the intraoperative period. Total intravenous anesthesia was used in view of large tumor with mass effect. Bispectral index-titrated propofol, fentanyl, and rocuronium infusion were used to maintain adequate depth of anesthesia and muscle relaxation respectively. Surgery was eventful and duration was around 5 hours with a blood loss of around $2 \mathrm{~L}$ which was replaced with five units packed red blood cells, five units fresh frozen plasma, and five units platelets concentrate. No inotropes or

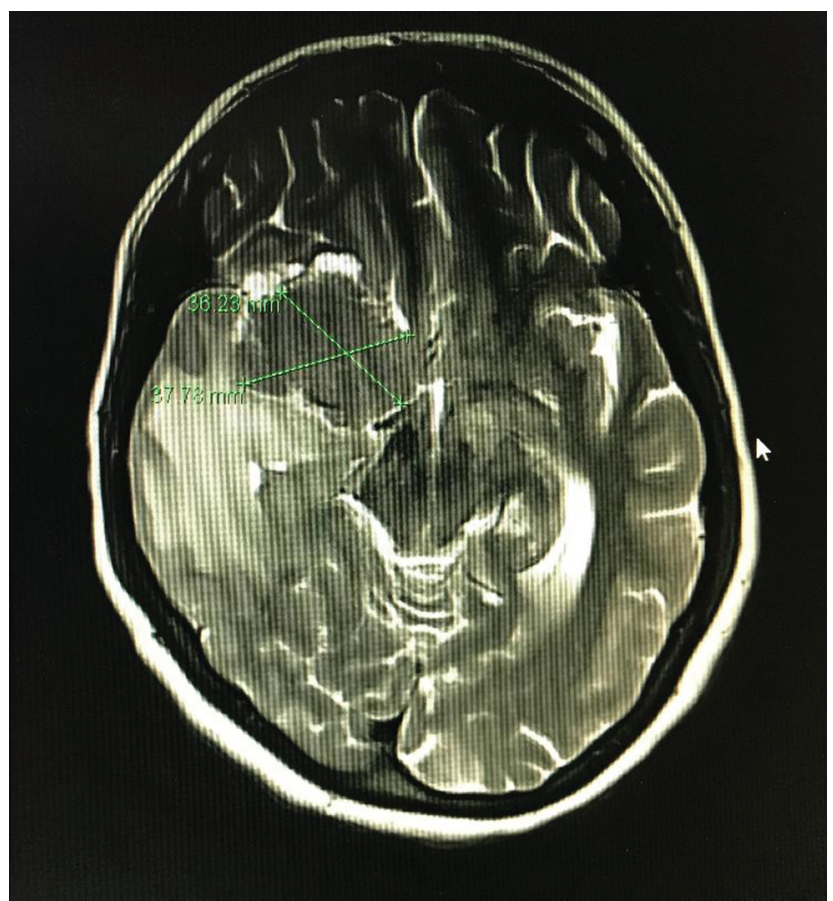

Fig. 1 Preoperative magnetic resonance imaging of the patient showing sphenoid wing meningioma.
(C2020. Indian Society of Neuroanaesthesiology and Critical Care. This is an open access article published by Thieme under the terms of the Creative Commons Attribution-NonDerivative-NonCommercial-License, permitting copying and reproduction so long as the original work is given appropriate credit. Contents may not be used for commercial purposes, or adapted, remixed, transformed or built upon. (https://creativecommons.org/licenses/by-nc-nd/4.0/).

Thieme Medical and Scientific Publishers Pvt. Ltd. A-12, 2nd Floor, Sector 2, Noida-201301 UP, India 


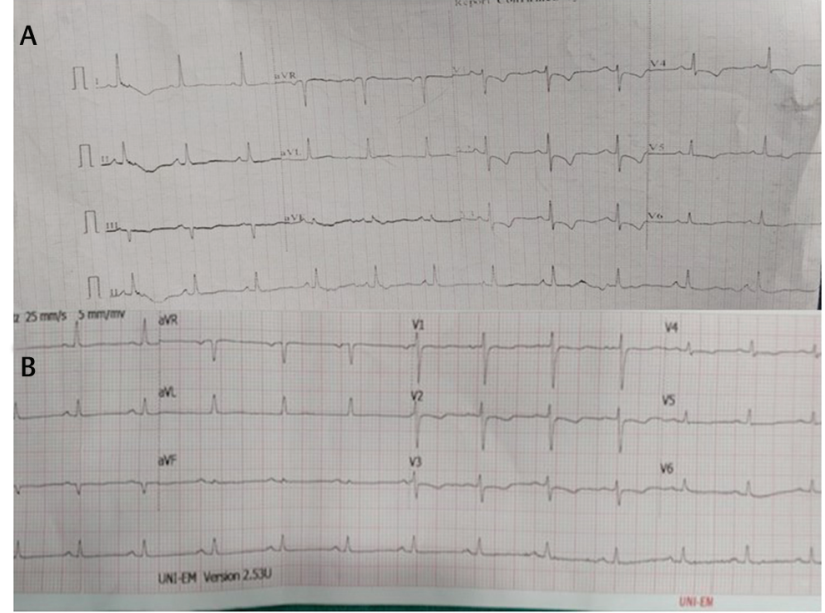

Fig. 2 (A) Preoperative ECG showing ST depression in lead II, V1-V5. (B) Postoperative ECG showing normalization of ECG changes. ECG, electrocardiogram.

vasopressors were required in the intraoperative period and patient was hemodynamically stable throughout the surgery except for the ECG changes. Postoperative elective ventilation was planned in view of massive blood loss, prolonged surgery, and cerebral edema. Patient was weaned and extubated on first postoperative day (POD). Patient was followed up for 7 days. Serial 12-lead ECG was recorded on POD 1-7, which revealed gradual normalization of ST-T changes and T wave inversions. - Fig. 2B shows the ECG on the 7th day, which depicted complete resolution of abnormal ECG changes. The commonly reported findings are ST elevation with T inversion; however, in our case, we found ST depression and T inversion. The ECG findings were not associated with any signs and symptoms but as a part of screening, Trop T and $2 \mathrm{D}$ echo were done. Trop $\mathrm{T}$ was negative and echocardiography was normal. As the patient was stable hemodynamically and had no complains of chest pain and stable condition postoperatively, she was not subjected to serial Trop T, serial 2D echo, or invasive cardiac angiogram. Invasive cardiac angiogram should be reserved for cases of abnormal echocardiography showing regional motion abnormalities. Currently, there are no guidelines particularly for neurosurgical patients; however, some studies have impressed upon the use of ECG, serum biomarkers, and echocardiography for preoperative evaluations. ${ }^{4}$ The American College of Cardiology (ACC)/American Heart Association (AHA) task force has reserved invasive tests such as cardiac angiogram for grossly abnormal echocardiograph like regional wall motion abnormalities or abnormal septal motions which were absent in our case. In absence of abnormalities in echocardiography, no further testing is recommended. ${ }^{5}$

From the this report we want to emphasize that neurological insult has a bearing on ECG which can have manifestations or may remain subtle. In our case, the ECG changes started resolving postoperatively and complete normalization was seen by the end of first week. ECG changes in neurosurgical patient can be due to organic heart disease or secondary to neurological insult. The later tend to resolve with treatment of neurological insult. Cardiac status of the patient should be evaluated from relevant history, clinical examination, biomarkers, and echocardiography. Early treatment of neurological insult should help in resolution of ECG changes. Documentation of persistence/resolution of such ECG changes may be helpful while evaluating the patient in future.

\section{Conflict of Interest}

None declared.

\section{Reference}

1 Manikandan S. Heart in the brain injured. J Neuroanaesth Crit Care 2016;3:S:12-S154.

2 Yogendranathan N, Herath HM, Pahalagamage SP, Kulatunga A. Electrocardiographic changes mimicking acute coronary syndrome in a large intracranial tumour: A diagnostic dilemma. BMC Cardiovasc Disord 2017;17(1):91

3 Bhagat H, Narang R, Sharma D, Dash HH, Chauhan H. ST elevation-an indication of reversible neurogenic myocardial dysfunction in patients with head injury. Ann Card Anaesth 2009;12(2):149-151

4 Lieb K, Selim M. Preoperative evaluation of patients with neurological disease. Semin Neurol 2008;28(5):603-610

5 Fleisher LA, Fleischmann KE, Auerbach AD, et al. 2014 ACC/AHA guideline on perioperative cardiovascular evaluation and management of patients undergoing noncardiac surgery: executive summary: a report of the American College of Cardiology/American Heart Association Task Force on Practice Guidelines. Circulation 2014;130(24):2215-2245 \\ Intraoperative Predissection Aneurysm Rupture: No Less than a Catastrophe!
}

\author{
Nayani Radhakrishnan ${ }^{1}$ Rajendra S. Chouhan ${ }^{2}$ Indu Kapoor ${ }^{2}$ Charu Mahajan²
}

\footnotetext{
${ }^{1}$ Department of Anaesthesia and Critical Care, Army Hospital Research and Referral, New Delhi, India

2Department of Neuroanaesthesiology and Critical Care, All India Institute of Medical Sciences (AlIMS), New Delhi, India
}

Address for correspondence Charu Mahajan, MD, DM, Department of Neuroanaesthesiology and Critical Care, Neurosciences Centre, All India Institute of Medical Sciences (AlIMS), New Delhi 110029, India (e-mail: charushrikul@gmail.com). 